\title{
Thermodynamic Calculation Study on Solid Solutions' Stabilities of Au-Pd-M (M=Mo, Y, Zr)
}

\author{
Wang Saibei ${ }^{\mathrm{a}^{\star}}$, Xie Ming $^{\mathrm{b}}$, Zhang Jiming ${ }^{\mathrm{c}}$, Wang Song ${ }^{\mathrm{d}}$, Hu Jieqiong ${ }^{\mathrm{e}}$, Liu \\ Manmen ${ }^{f}$ and Li Aikun ${ }^{g}$
}

State Key Laboratory of Advanced Technologies for Comprehensive Utilization of Platinum Metals, Sino-platinum Metals Co. Ltd., Institute of Precious Metals, Kunming 650106, China

a*77481311@163.com, bpowder@ipm.com.cn, ćzjm_zane@163.com,

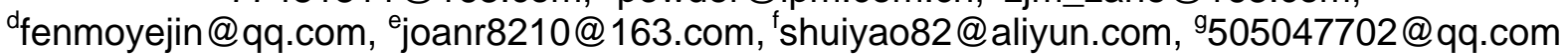

Keywords: Au-Pd-M; first-principle; solid solution; phase stability

Abstract. According to the first-principle based on density functional theory, the stability of the solid solutions of Au-Pd-M (M=Mo,Y,Zr) alloy systems were calculated. The results show that, adding the same percentage content, $1 \%$ Mo doped in Au-Pd solid solution is the most stable phase structure. When doped with Y and Zr, they are likely to react with Au-Pd to form the corresponding intermetallic compounds.

\section{Introduction}

Precious metals alloy materials are the key basic materials for electron, electrics and chemical industries [1-3], the further research on the phase structures and properties will be beneficial to improve the basic researching system of precious metals alloy materials and provide theoretical support to their applications. In this article, the representative precious alloy system Au-Pd-M $(\mathrm{M}=\mathrm{Mo}, \mathrm{Y}, \mathrm{Zr}$ ) is chosen for study. The relationship between phase structures and phase composition will be investigated. The optimization design theoretical system of compositions, phase structures and properties of rare and precious metals alloys will be established. All these study will lay a foundation of theory and application to the design and fabrication of the new precious metals and rare metals alloy with high properties.

\section{Experimental}

Phase diagram show that Au-Pd alloy is continuous solid solution in high temperature zone under the solidus. Therefore, a solid solution model with $\mathrm{Au}$ as solvent and $\mathrm{Pd}$ as solute was constructed, as shown in Fig.1. The corresponding models were built with $\mathrm{M}(\mathrm{M}=\mathrm{Mo}, \mathrm{Y}, \mathrm{Zr})$ content $1 \%, 2 \%$ and $5 \%$ (mass percent) according to the quality of composition proportion. Doping $\mathrm{M}$ could be achieved through modifying the atoms of digits.

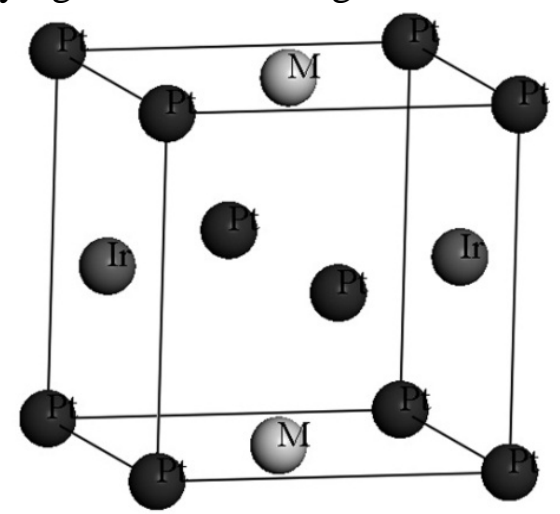

Fig.1. Crystal structure of Au-Pd-M

All calculations are performed with Cambridge serial total energy package (CASTEP) code based on the first-principles method within the framework of density function theory 
(DFT).Vanderbilt-type ultrasoft pseudo-potentials (USPP) are employed to describe the electron-ion interactions. The exchange and correlation terms are described with the local-density approximation (LDA)[4] parameterized by PERDEW and ZUNGER[5] and the generalized gradient approximation (GGA) in the scheme of Perdew-Wang 91 (PW91)[6]. It needs to be mentioned that the used USPP should be compatible with the special type of LDA and GGA. In the calculations, we use $6 \times 6 \times 6$ mesh of special k-points and the kinetic energy cutoff (Ecut) of $340 \mathrm{eV}$ for both the LDA calculations and the GGA calculations, using SCF error of $2.0 \times 10-6 \mathrm{eV}$, in addition, all of the selected other parameters being adequate accuracy.

\section{Results}

Energetic stability of Au-Pd-M (M=Mo, Y, Zr) system. The calculated cohesive energies and enthalpies of formation are presented in Table 1. It can be seen from Table 2 that with the increasing of doping element, the calculated cohesive energies and enthalpies of formation of Au-Pd-M (M= Mo, Y, Zr) system are becomes higher. This implies that the low content of doping element, the solid solutions is more easily formed, and the formed solid solutions are more stable.

Table 1 Cohesive energies and formation enthalpies of Mo, Y, Zr with different composition ratio

\begin{tabular}{rrcc}
\hline \multicolumn{2}{c}{ Percentage composition } & E[eV/atom $]$ & $\mathrm{H}[\mathrm{eV} /$ atom $]$ \\
\hline \multirow{2}{*}{ Mo } & $1 \%$ & -7.68 & -10.58 \\
& $2 \%$ & -7.53 & -10.37 \\
& $5 \%$ & -7.16 & -9.87 \\
$1 \%$ & -6.71 & -9.58 \\
$\mathrm{Y}$ & $2 \%$ & -6.60 & -9.43 \\
& $5 \%$ & -6.22 & -8.89 \\
& $1 \%$ & -7.27 & -10.31 \\
$\mathrm{Zr}$ & $2 \%$ & -7.13 & -10.11 \\
& $5 \%$ & -6.74 & -9.56 \\
\hline
\end{tabular}

Meanwhile, compared with three doping elements Mo, Y, Zr in Au-Pd solid solution, we found that with the same percentage of doping, the cohesive energies and enthalpies of formation of Au-Pd-Mo solid solution are less than the other two elements doping, which indicates that Au-Pd-Mo is formed more easily than Au-Pd-Zr and Au-Pd-Y, and the formed Au-Pd-Mo is more stable than Au-Pd-Zr and Au-Pd-Y.

Bonding characters of solid solutions. This section focuses on the bonding characteristics of Au-Pd solid solution doped with three different elements, electron occupation and electronic transfers on each electron orbit of Au-Pd-M (M=Mo, Y, Zr) solid solutions are showing in Table 2, Table 3 and Table 4. It can be seen from the following three tables, the electrons at valence electron orbit of each element have changed, but the whole crystal cell maintain electro-neutrality, that is hybridization happened between electrons. It also can be found in the tables that with the same percentage of doping elements Mo, Y, Zr in Au-Pd solid solution, the electronic transfer number of $\mathrm{Y}$ element is the largest, followed by the $\mathrm{Zr}$ element, and the electron transfer number of Mo element is the smallest. This also means that $\mathrm{Y}$ and $\mathrm{Zr}$ are more easily react with elements in the Au-Pd solid solutions and generates the corresponding alloy.

Electronic structures of solid solutions. As mentioned above, compared with three doping elements Mo, Y, Zr and mass percentage of doping elements in Au-Pd solid solution, we found that the most stable structures is the $1 \%$ of trace alloy doped structure. So, here we discuss the phase stability of $1 \%$ Mo, Y, Zr doped in Au-Pd solid solution in terms of electronic structures. The valence electron of Pd and Au, Mo, Y, Zr are: Pd: 4s24p64d10, Au: 4p65d106s1, Mo: 4p64d55s1, Y: 4p64d15s2, Zr:4p64d25s2, obviously, they are the transition metals, whose d electron orbital is not fully occupied and liable to occur electronic transfers. 
Table 2 Electron occupation and electronic transfers on each electron orbit of Au-Pd-Mo

\begin{tabular}{|c|c|c|c|c|c|c|c|}
\hline \multirow{2}{*}{$\begin{array}{l}\text { Percentage } \\
\text { composition }\end{array}$} & \multicolumn{3}{|c|}{$\mathrm{s}$} & $\mathrm{p}$ & \multicolumn{2}{|c|}{ d } & \multirow{2}{*}{$\begin{array}{c}\text { Electronic } \\
\text { transition }\end{array}$} \\
\hline & $4 s$ & $5 s$ & $6 s$ & $4 p$ & $4 d$ & $5 d$ & \\
\hline Mo & - & 2.42 & - & 6.66 & 4.93 & - & -0.02 \\
\hline $\mathrm{Au}$ & - & - & 0.83 & 0.66 & - & 9.51 & 0.00 \\
\hline $\mathrm{Pd}$ & 0.27 & - & - & 0.37 & 9.33 & - & 0.02 \\
\hline \multicolumn{8}{|c|}{ Table 3 Electron occupation and electronic transfers on each electron orbit of Au-Pd-Y } \\
\hline \multirow{2}{*}{$\begin{array}{l}\text { Percentage } \\
\text { composition }\end{array}$} & \multicolumn{3}{|c|}{ 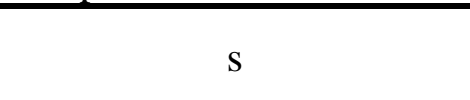 } & $\mathrm{p}$ & \multicolumn{2}{|c|}{ d } & $\begin{array}{l}\text { Electronic } \\
\text { transition }\end{array}$ \\
\hline & $4 \mathrm{~s}$ & $5 s$ & $6 s$ & $4 \mathrm{p}$ & $4 \mathrm{~d}$ & $5 \mathrm{~d}$ & \\
\hline $\mathrm{Y}$ & - & - & 2.08 & 6.22 & 1.52 & - & 1.19 \\
\hline $\mathrm{Au}$ & - & - & 0.99 & 0.79 & - & 9.58 & -0.72 \\
\hline $\mathrm{Pd}$ & 0.99 & - & - & 0.64 & 9.38 & & -0.47 \\
\hline Table 4 El & occup & and el & nic tra & rs on $\epsilon$ & lectro & $\mathrm{t}$ of $A$ & $\mathrm{Pd}-\mathrm{Zr}$ \\
\hline \multirow{2}{*}{$\begin{array}{c}\text { Percentage } \\
\text { composition }\end{array}$} & \multicolumn{3}{|c|}{$\mathrm{S}$} & $\mathrm{p}$ & \multicolumn{2}{|c|}{ d } & $\begin{array}{l}\text { Electronic } \\
\text { transition }\end{array}$ \\
\hline & $4 s$ & $5 s$ & $6 s$ & $4 p$ & $4 d$ & $5 d$ & \\
\hline $\mathrm{Zr}$ & - & 2.27 & - & 6.55 & 2.43 & - & 0.75 \\
\hline $\mathrm{Au}$ & - & - & 0.95 & 0.76 & - & 9.52 & -0.45 \\
\hline $\mathrm{Pd}$ & 0.39 & - & - & 0.58 & 9.32 & - & -0.30 \\
\hline
\end{tabular}

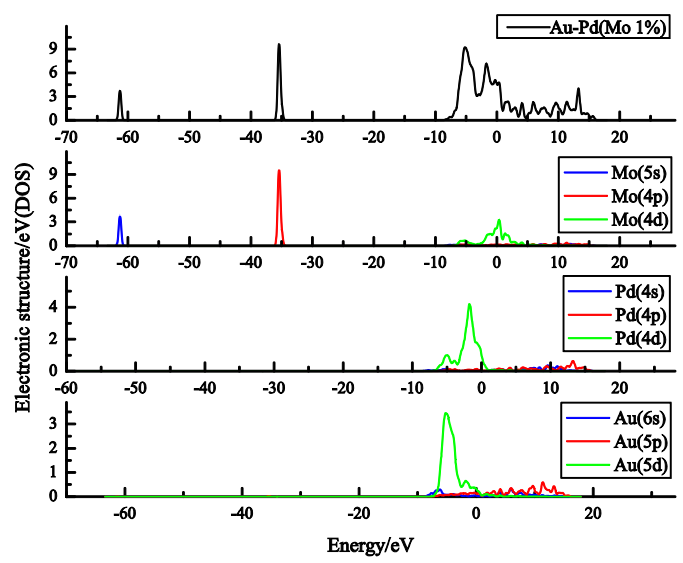

(a) Au-Pd-Mo(1\%)DOS

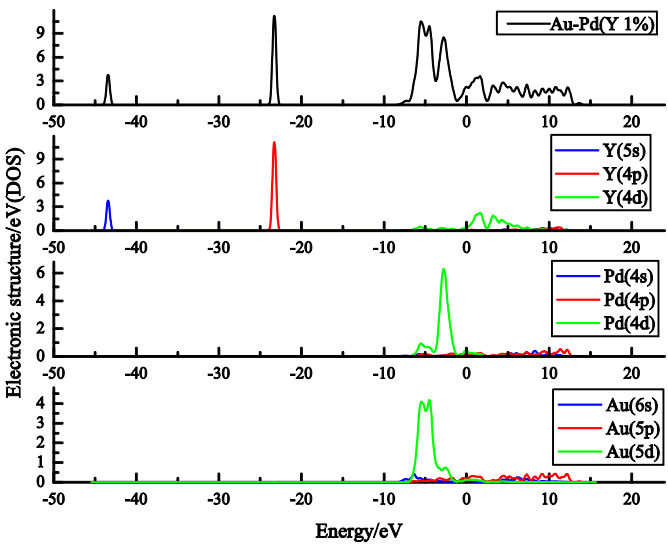

(b) Au-Pd-Y(1\%)DOS

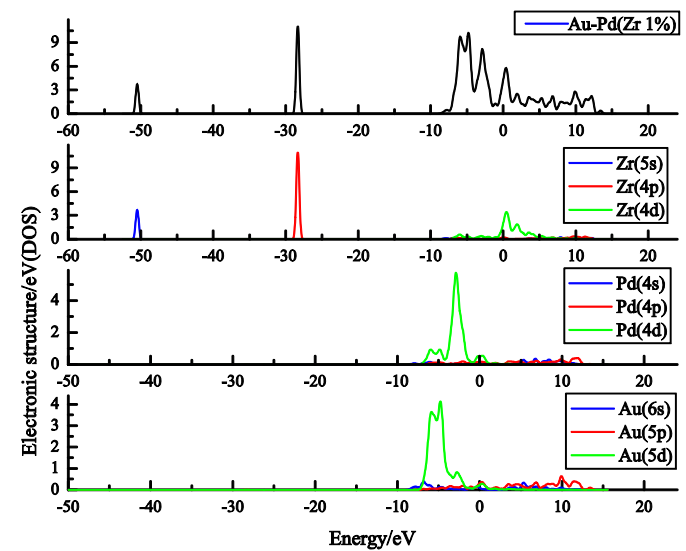

(c) Au-Pd-Zr(1\%)DOS

Fig. 2. The total and partial density of states at equilibrium lattice constants for $1 \% \mathrm{Mo}, \mathrm{Y}$, $\mathrm{Zr}$ doped in Au-Pd solid solution 
The total and partial density of states at equilibrium lattice constants for $1 \% \mathrm{Mo}$, Y, Zr doped in Au-Pd solid solution are showing in Fig.2. It can be seen from Fig.2 that the main contributions to the DOS near the Fermi level of Au-Pd-Mo solid solution come from the 5d-states of Au and the 4d-states of Pd, Mo respectively; the main contributions to the DOS near the Fermi level of Au-Pd-Y solid solution come from the 5d-states of Au and the 4d-states of Pd, Y respectively; the main contributions to the DOS near the Fermi level of Au-Pd-Zr solid solution come from the 5d-states of $\mathrm{Au}$ and the 4d-states of $\mathrm{Pd}$ and $\mathrm{Zr}$ respectively. This implies that the electron transfer only occurs at d electron orbit near Fermi level, and the appropriate chemical bond formed along d-d direction.

As compared with Au-Pd-Mo system, the main peaks of Au-Pd-Y and Au-Pd-Zr system, on the one hand, split into two peaks, on the other hand, the peak of Pd offset to the left, and $\mathrm{Au}$ is offset to the right, which means that doping $\mathrm{Y}$ and $\mathrm{Zr}$ causes violent electronic hybridization near the Fermi surface, reacting to form the corresponding intermetallic compounds.

\section{Conclusions}

(1) Pd-Ru-Mo is formed more easily than Pd-Ru-Zr and Pd-Ru-Y, and the formed Pd-Ru-Mo is more stable than Pd-Ru-Zr and Pd-Ru-Y.

(2) When doped with $\mathrm{Y}$ and Zr, they are likely to react with Au-Pd to form the corresponding intermetallic compounds.

\section{Acknowledgment}

In this paper, the research was sponsored by the National Science \& Technology Pillar Program(Project No. 2012BAE06B05), the National Natural Science Foundation of China(Project No. u0837601,u1302272), the Science and Technology Innovation Team of Kunming (Project No. 20120101AR070005)and the Innovation Team of Yunnan Province(Project No. 2012HC027).

\section{References}

[1] Jones F.H., Teeth and bones: applications of surface science to dental materials and related biomaterials, Surface Science Reports., 2001, 42 (3-5): 75.

[2] Hsin Y.L., and Joel D.B., Changes in the surface oxide composition of Co-Cr-Mo implant alloy by macrophage cells and their released reactive chemical species, Biomaterials, 2004,25 (7-8): 1233.

[3] Darling A.S., and Yorke J.M., The ruthenium-palladium system, Platinum Metals Review, 1960, 4 (3): 104.

[4] CEPERLEY D M, ALDER B J. Ground state of the electron gas by a stochastic method [J]. Phys Rev Lett, 1980, 45(7): 566-569.

[5] PERDEW J P, ZUNGER A. Self-interaction correction to density-functional approximations for many-electron systems [J]. Phys Rev B, 1981, 23(10): 5048-5079.

[6] PERDEW J P, CHEVARY J A, VOSKO S H, JACKSON K A, FIOLHAIS C. Atoms, molecules, solids, and surfaces: Applications of the generalized gradient approximation for exchange and correlation [J]. Phys Rev B, 1992, 46(11): 6671-6687. 